\title{
Análisis de microdensidad vascular y factores de crecimiento en carcinoma oral de células escamosas.
}

\author{
Analysis of vascular microdensity and growth factors in oral squamous cell carcinoma. \\ Itzel Legorreta-Villegas, ${ }^{*}$ David Alonso Trejo-Remigio, ${ }^{\ddagger}$ Carla Monserrat Ramírez-Martínez, ${ }^{\ddagger}$ Javier Portilla-Robertson, ${ }^{\ddagger}$ \\ Elba Rosa Leyva-Huerta, ${ }^{\ddagger}$ Luis Fernando Jacinto-Alemán ${ }^{\ddagger}$
}

\section{RESUMEN}

Introducción: El carcinoma oral de células escamosas (COCE) es una neoplasia epitelial maligna que se presenta frecuentemente entre la quinta y sexta década de la vida. Su compleja patogénesis incluye el proceso de angiogénesis y la regulación del microambiente tumoral como mecanismos de progresión tumoral. Objetivo: Determinar la relación entre las variables clínicas e histológicas del COCE con la inmunoexpresión de VEGF, FGF-1, FGFR-1, TGFB-1, TGFBR-II y CD105. Material y métodos: Nueve casos de COCE; tres bien (BD), tres moderado (MD) y tres pobremente diferenciados (PD) obtenidos del Departamento de Patología y Medicina Bucal, División de Estudios de Postgrado e Investigación. Se aplicó la técnica de inmunohistoquímica por peroxidasa para identificar la expresión de VEGF, FGF-1, FGFR1, TGFB-1, TGFBR-II y CD105. El análisis de inmunoexpresión se realizó con el programa ImageJ. Se aplicó la prueba de Kruskal-Wallis y correlación de Spearman $(\mathrm{p}<0.05)$. Resultados: La inmunoexpresión de VEGF fue mayor en los COCE PD, FGFR-1 fue positivo en los BD, mientras que FGF, TGFB-1 y TGFBR-II fueron negativos. El análisis de microdensidad vascular (MVD) indicó mayor número de vasos CD105 positivos en los carcinomas BD, seguidos de los PD y MD. Conclusión: Considerando los resultados obtenidos podemos concluir que la angiogénesis es un fenómeno constante independiente del grado de diferenciación que durante el proceso de transformación de una neoplasia requerirá la formación de vasos sanguíneos y que este proceso puede ser modulado por factores de crecimiento tales como los analizados en este trabajo.

Palabras clave: Factores de crecimiento, microdensidad vascular, VEGF, FGFR-1.

\section{ABSTRACT}

Introduction: Oral squamous cell carcinoma (OSCC) is a malignant epithelial neoplasm that frequently occurs between the fifth and sixth decade of life. Its complex pathogenesis includes the angiogenesis process and the regulation of the tumor microenvironment as mechanisms of tumor progression. Objective: To determine the relationship between the clinical and histological variables of OSCC with the immunoexpression of VEGF, FGF-1, FGFR-1, TGFB1, TGFBR-II and CD105. Material and methods: Nine cases of OSCC; three well (WD), three moderate (MD) and three poorly differentiated (PD) obtained from the Oral Medicine and Pathology Department, Division of Graduate Studies and Research. The peroxidase immunohistochemistry technique was performed to identify the expression of VEGF, FGF-1, FGFR-1, TGFB-1, TGFBR-II and $C D 105$. The immunoexpression analysis was performed with the ImageJ software. The Kruskal-Wallis and Spearman correlation test were performed $(p<0.05)$. Results: VEGF immunoexpression was higher in PD OSCC, while FGFR-1 was predominantly positive in WD; FGF, TGFB-1 and TGFBR-II were negative. Vascular microdensity analysis (MVD) indicated a greater number of CD105 positive vessels in WD carcinomas, followed by PD and MD. Conclusion: Considering the results obtained, we can conclude that angiogenesis is a constant phenomenon independent of the degree of differentiation; that during the transformation process of a neoplasm it will require the formation of blood vessels and that this process can be modulated by growth factors such as those analyzed in this work.

Keywords: Growth factors, vascular microdensity, VEGF, FGFR-1.

\footnotetext{
* Cirujana Dentista.

₹ Profesor, Especialidad Patología y Medicina Bucal, División de Estudios de Postgrado e Investigación.
}

Facultad de Odontología, Universidad Nacional Autónoma de México.

Recibido: 26 de agosto de 2020. Aceptado para publicación: 17 de noviembre de 2020.

Citar como: Legorreta-Villegas I, Trejo-Remigio DA, Ramírez-Martínez CM, Portilla-Robertson J, Leyva-Huerta ER, Jacinto-Alemán LF. Análisis de microdensidad vascular y factores de crecimiento en carcinoma oral de células escamosas. Rev ADM. 2020; 77 (6): 287-294. https://dx.doi.org/10.35366/97616

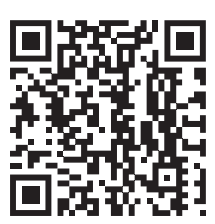




\section{INTRODUCCIÓN}

C áncer es un término que se usa para designar una enfermedad que puede presentarse en cualquier parte del organismo; se caracteriza por la proliferación anormal y autónoma de células epiteliales o mesenquimales, capaces de evadir la apoptosis e invadir localmente y a distancia otros órganos y tejidos, es decir, metastatizar. ${ }^{1,2}$ El carcinoma oral de células escamosas (COCE) es una neoplasia maligna epitelial. Es más frecuente en la quinta y sexta décadas de la vida, por lo general se asocia con factores de riesgo como el tabaquismo, el consumo de alcohol y la masticación de la nuez de betel en el subcontinente asiático. ${ }^{3}$

El cáncer bucal ocupa el sexto lugar de incidencia de neoplasias malignas en el mundo. Al menos 95\% de las neoplasias de la cavidad bucal son carcinomas epidermoides. ${ }^{1,4}$ Datos de Globocan en 2018 indicaron que la prevalencia a cinco años para cáncer oral y de labio es de 913,514 casos, lo que representa 12.0 por cada 100,000 habitantes. En México la prevalencia a cinco años fue de 5,647 casos, lo que representa 4.3 casos por cada 100,000 habitantes. ${ }^{5}$ Además de los factores de riesgo clásicos (tabaco y alcohol), el virus del papiloma humano (subtipos 16 y 18) también ha sido considerado. ${ }^{6}$

Su carcinogénesis es sumamente compleja, ya que pueden observarse afecciones en oncogenes, genes supresores tumorales, regulación de la apoptosis, el reloj biológico, angiogénesis, invasión y metástasis, entre otras. ${ }^{7,8}$ Dentro de los oncogenes, los factores de crecimiento son moléculas secretadas que promueven o inhiben la mitosis o diferenciación celular. Su función como oncogenes puede derivar de su sobreexpresión o sobreactivación propiciando el desarrollo del cáncer. ${ }^{9}$

El factor de crecimiento transformate- $\beta$ (TGF- $\beta$ ) desempeña un papel importante en el microambiente extracelular, proliferación, diferenciación, apoptosis y migración celular. ${ }^{10}$ Tras la unión del ligando se une al receptor T $\beta R$ II, la formación de complejo heterotetramérico conduce a la señalización que induce la sobreproliferación e inhibición de la apoptosis en la oncogénesis. ${ }^{11}$

El factor de crecimiento fibroblástico 1 (FGF-1) es un factor de crecimiento que tiene una amplia distribución en tejidos normales y neoplásicos. Este factor de crecimiento ejerce sus actividades biológicas a través de receptores de FGF de superficie celular de alta afinidad. ${ }^{12}$ Osada y colaboradores han reportado la importancia de FGF1 y su receptor en el proceso de transición epitelio mesénquima, proceso sumamente importante en la trasformación maligna, ya que al ser inhibida su señalización restaura el fenotipo epitelial de las células neoplásicas. ${ }^{13}$
Otra función biológica importante que participa en el proceso carcinogénico es la angiogénesis, que se define como la formación de nuevos vasos sanguíneos a partir de vasos preexistentes. ${ }^{14}$ Es un proceso que implica la migración y proliferación de células endoteliales, la formación y organización de estructuras tubulares que con el tiempo se unirán, para finalmente madurar en vasos sanguíneos estables. ${ }^{15}$ Los factores proangiogénicos y factores de crecimiento son los responsables de este proceso. Algunos de ellos serán considerados indicadores o biomarcadores que relacionan a la angiogénesis con alguna característica biológica de la neoplasia. La evaluación de la angiogénesis tumoral puede realizarse de manera histológica, radiológica y serológica. La evaluación histológica es la más común y con ella se efectúa la medición de la angiogénesis y la densidad microvascular (MVD). ${ }^{16}$ La MVD se define como el número de vasos pequeños en una determinada área tumoral. ${ }^{17}$ Se evalúa observando áreas seleccionadas al azar contando las zonas de mayor densidad vascular, lo cual puede ser correlacionado con la progresión y el pronóstico de varios tipos de tumores malignos. ${ }^{17} \mathrm{El}$ objetivo de este trabajo fue determinar la correlación de la inmunoexpresión de VEGF, FGF-1, FGFR-1, TGFB-1, TGFBR-II; y MVD a través de CD105 con el grado de diferenciación del COCE.

\section{MATERIAL Y MÉTODOS}

Población: nueve casos de COCE (tres para cada grado de diferenciación) obtenidos del Archivo de Diagnóstico Histopatológico del Departamento de Patología y Medicina Bucal. Las muestras fueron seleccionadas aleatoriamente, eligiendo sólo los casos que tuvieran los datos clínicos demográficos completos (edad, sexo y localización anatómica) y presentaran suficiente tejido para su estudio inmunohistoquímico. Acorde con el aviso de privacidad de la Facultad de Odontología, UNAM, los datos obtenidos fueron empleados con la finalidad de difundir el conocimiento científico, respetando el anonimato del paciente y expediente clínico. ${ }^{18}$

Análisis histopatológico: se realizó una tinción con hematoxilina y eosina (HyE). El análisis y confirmación del diagnóstico histopatológico fue efectuado por dos patólogos bucales.

Ensayo de inmunohistoquímica: El procedimiento se realizó según lo reportado. ${ }^{19}$ Se hicieron nueve cortes a $4 \mu \mathrm{m}$ de cada caso seleccionado, colocándolos sobre laminillas previamente sialinizadas. Se realizó el proceso de desparafinación y rehidratación en inmersiones en 
xilol y alcohol de manera convencional. Posteriormente se procedió a recuperar los antígenos utilizando un buffer de citratos al $0.01 \mathrm{M}$ a baño maría en horno de microondas a $700 \mathrm{~W}$ durante cuatro minutos. Se hicieron tres lavados con buffer de fosfato (PBS) de tres minutos cada uno, para luego inhibir la actividad de la peroxidasa endógena con peróxido de hidrógeno al 3\% (Amresco, 7722-84-1, Radnor, PA, USA) durante 20 minutos. Se lavó con PBS y posteriormente se incubó con PBS-albúmina al 1\% (Amresco, 97061-422, Radnor, PA, USA) y Tritón x-100 al 1\% (Amresco, 97062-208, Radnor, PA, USA) durante 20 minutos cada uno. Se hicieron tres lavados de PBS de tres minutos. Se incubó toda la noche con los anticuerpos primarios VEGF (sc-7269), TFGB1 (sc-146), FGF (sc-55520), FGFR-1 (sc-57132), TGFBRII (sc-17791) y CD105 (sc-20632); todos de la marca Santa Cruz Biotecnology (Santa Cruz Biotechnology, Dallas, TX, USA), en una dilución 1:200. Como control negativo se sustituyó el anticuerpo con PBS.

Terminada la incubación se lavó con TBS (Solución Tris buffer) por tres minutos en tres ocasiones. Posteriormente se incubó con Super-Enhancer (BioGenex, QD430-XAKE, Fremont, CA) durante 20 minutos, para después lavar con TBS. Se incubó con biotin-link HRP (BioGenex, QD430-XAKE) por 30 minutos. Se lavó con TBS y enseguida se reveló con diaminobencidina (DAB, BioGenex, QD430-XAKE) durante cinco minutos. Se lavó con agua durante tres minutos, contratiñendo con hematoxilina de Harris durante dos minutos. Nuevamente se lavó con agua corriente para después realizar la deshidratación y aclarado en inmersiones de alcohol y xilol para montar el cubreobjetos con resina hidrofóbica.

Análisis de inmunoexpresión: para cada caso de COCE se obtuvieron cuatro fotomicrografías a 400 aumentos con el microscopio Leica DM750 y el software programa Leica Las-Ez versión 3.2.0. En cada fotomicrografía fue cuantificada la intensidad y la proporción de células positivas mediante el software Image $(\mathrm{NIH}$, Bethesda MD, USA). La intensidad de inmunoexpresión fue expresada en unidades ópticas (uo). La proporción fue calibrada en el software de la siguiente manera: 0 ) ausencia, 1) 0 a $10 \%$, 2) 11 a $50 \%$ y 3) superior a $50 \%$.

Análisis de MVD: se tomaron tres fotografías a 200 aumentos de cada caso para el marcador CD105. En cada campo óptico se realizó el conteo de vasos sanguíneos.

Análisis estadístico: para la cantidad de células positivas, intensidad de inmunoexpresión y MVD se obtuvieron media y desviación estándar. Se realizó el análisis estadístico no paramétrico Kruskal-Wallis y prueba de correlación de Spearman en relación con el grado de diferenciación considerando una $p<0.05$ como significativa.

\section{RESULTADOS}

\section{Análisis clínico-histológico}

De los nueve casos seleccionados del departamento de patología, medicina bucal y maxilofacial, la edad promedio fue de $55.3 \pm 11.7$ años, con rango de edad de 39 a 75 años. El sexo femenino fue más frecuente

Tabla 1: Datos clínico-demográficos.

\begin{tabular}{lcccc}
\hline Grado de diferenciación & Caso & Edad & Género & Localización \\
\hline Bien diferenciado & BD1 & 57 & Femenino & Borde lateral de la lengua \\
& BD2 & 52 & Femenino & Borde lateral de la lengua \\
& BD3 & 58 & Masculino & Mucosa yugal \\
Moderadamente diferenciado & MD1 & 65 & Femenino & Piso de boca \\
& MD2 & 39 & Femenino & Mandíbula \\
Pobremente diferenciado & MD3 & 39 & Femenino & Mandíbula \\
& PD1 & 75 & Femenino & Borde lateral de lengua \\
& PD2 & 62 & Masculino & Vestíbulo y hemipaladar izquierdo \\
& PD3 & 51 & Masculino & Borde lateral de lengua \\
\hline
\end{tabular}

$\mathrm{BD}$ = bien diferenciado, $\mathrm{MD}$ = moderadamente diferenciado, $\mathrm{PD}$ = pobremente diferenciado . 

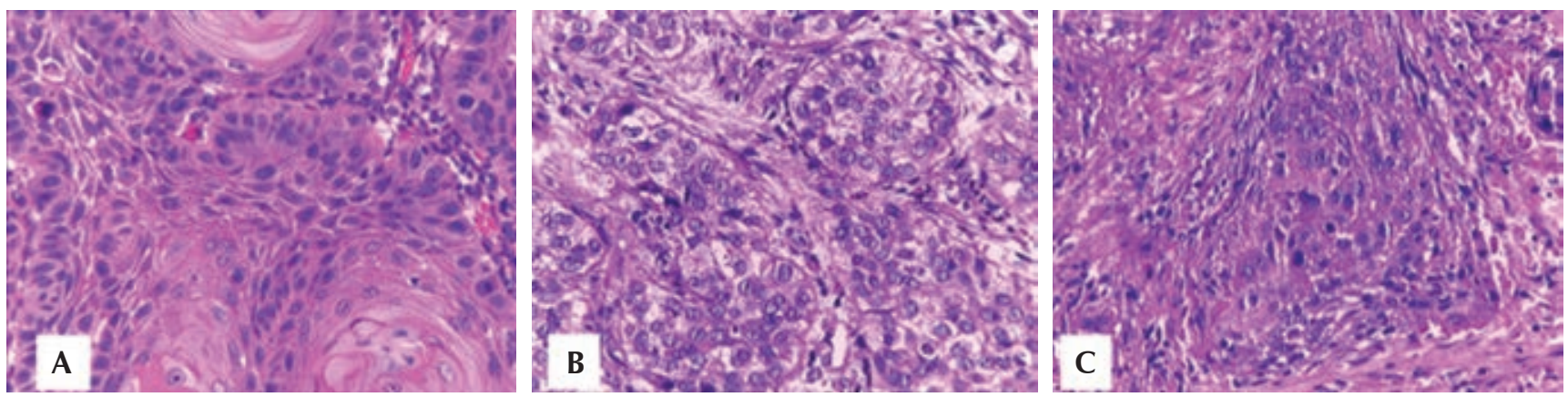

Figura 1: Características histopatológicas. A) Bien diferenciado. B) Moderadamente diferenciado y C) Pobremente diferenciado. 400 x.

(seis casos). Respecto a su localización anatómica, cuatro casos se presentaron en lengua, dos en mandíbula, uno en piso de boca, uno en mucosa yugal y uno en paladar (Tabla 1).

El análisis histológico mostró características propias de cada grado de diferenciación; brevemente en los COCEs bien diferenciados se observó proliferación neoplásica de estirpe epitelial compuesta por células poligonales que forman perlas de queratina; los carcinomas moderadamente diferenciados se caracterizaron por formación de islas de células con pleomorfismo células y nuclear y reforzamiento de la membrana basal, mientras que en el pobremente diferenciado predominó la atipia celular (Figura 1).

\section{Análisis de inmunoexpresión}

En la inmunoexpresión de VEGF se observó mayor intensidad de inmunoexpresión en los carcinomas PD, pero mayor proporción de células positivas en los BD (Figura 2 A-C). Respecto a la inmunoexpresión de FGFR1 , se observó mayor intensidad entre los carcinomas $\mathrm{BD}$ y $\mathrm{PD}$ que en los MD (Figura 2 D-F); con relación a la proporción de células positivas este marcador fue abundante en los tres grados de diferenciación. La inmunoexpresión de FGF-1, TGFB-1 y TGFBR-II fue negativa en los tres grados de diferenciación (Tabla 2; Figura 2 G-I).

\section{Análisis de MVD}

El conteo de vasos sanguíneos mediante el marcador CD105 indicó un ligero predominio en el promedio de vasos en los carcinomas BD con 10.5 vasos, 7.4 vasos en los MD y 9.5 vasos en los PD. Nuestro análisis estadístico no reportó asociaciones significativas.

\section{DISCUSIÓN}

En relación con su epidemiología y calidad de vida de los pacientes que lo padecen, convierte al cáncer oral en un problema de salud pública a nivel mundial, el cual debe ser investigado por múltiples enfoques, incluido el estudio celular o molecular. Se ha descrito que el COCE se presenta con más frecuencia en pacientes de la quinta y sexta décadas de la vida. ${ }^{3,20}$ La edad promedio de nuestros pacientes fue de $55.3 \pm 11.7$ años, lo cual concuerda con lo reportado. Este fenómeno puede tener relación con el tiempo de exposición ante los factores de riesgo ambientales clásicos como el tabaco y el alcohol; sin embargo, también existen reportes que sugieren que el COCE se puede presentar en pacientes más jóvenes, dejando abierta la discusión sobre la búsqueda de otros factores de riesgo. ${ }^{21}$

Respecto a la distribución por género, seis casos (67\%) correspondían a mujeres, mientras que tres casos (33\%) eran hombres. Tradicionalmente se ha considerado que el género masculino se ve más afectado; no obstante, se ha reportado mayor frecuencia en el género femenino. ${ }^{21}$ Este fenómeno puede ser debido a cuestiones conductuales tales como mayor exposición a tabaco y alcohol por el género femenino. De acuerdo con lo descrito en la literatura, la zona anatómica afectada con más frecuencia es la lengua, lo cual coincide con nuestros resultados. Se considera que esta zona anatómica es susceptible, ya que es la primera en entrar en contacto con los factores de riesgo ambientales tales como tabaco y alcohol, y por ser donde se reportan con mayor frecuencia lesiones potencialmente malignas y displasia. ${ }^{21-23}$ Esta característica reafirma el papel fundamental que tiene la exploración oral total con énfasis en zonas de riesgo como la lengua cuando los pacientes tienen antecedentes de tabaquismo o de consumo de alcohol. 
En nuestro análisis inmunohistoquímico se seleccionaron factores de crecimiento con mayor efecto teórico en células del estroma que en células del parénquima tumoral. Se ha reportado ampliamente el efecto que tienen factores de crecimiento tales como los miembros de la familia erbB en la transformación y crecimiento tumoral. ${ }^{24,25}$ No obstante, indagar qué pasa con otras moléculas principalmente relacionadas con el estroma, podría aportar información adicional sobre la conducta biológica del COCE y más aún si estos factores se pueden relacionar directa o indirectamente con la angiogénesis. Nuestros resultados sólo indicaron inmunoexpresión positiva para VEGF y FGFR-1. La inmunoexpresión de
VEGF fue mayor en los COCE pobremente diferenciados, seguidos de los moderados y bien diferenciados, es decir, fue inversamente proporcional con el grado de diferenciación. Este fenómeno nos sugeriría que los carcinomas pobremente diferenciados cuentan con más herramientas para inducir angiogénesis mediante VEGF; no obstante, existen reportes en los que este fenómeno es un patrón inverso donde los carcinomas bien diferenciados son los de mayor inmunoexpresión. ${ }^{26}$ Esta controversia nos confirma la necesidad de más estudios relacionados, ya que, al ser considerado el candidato más potente para la inducción de la angiogénesis en el crecimiento tumoral, éste podría significar un blanco terapéutico a futuro. Otra
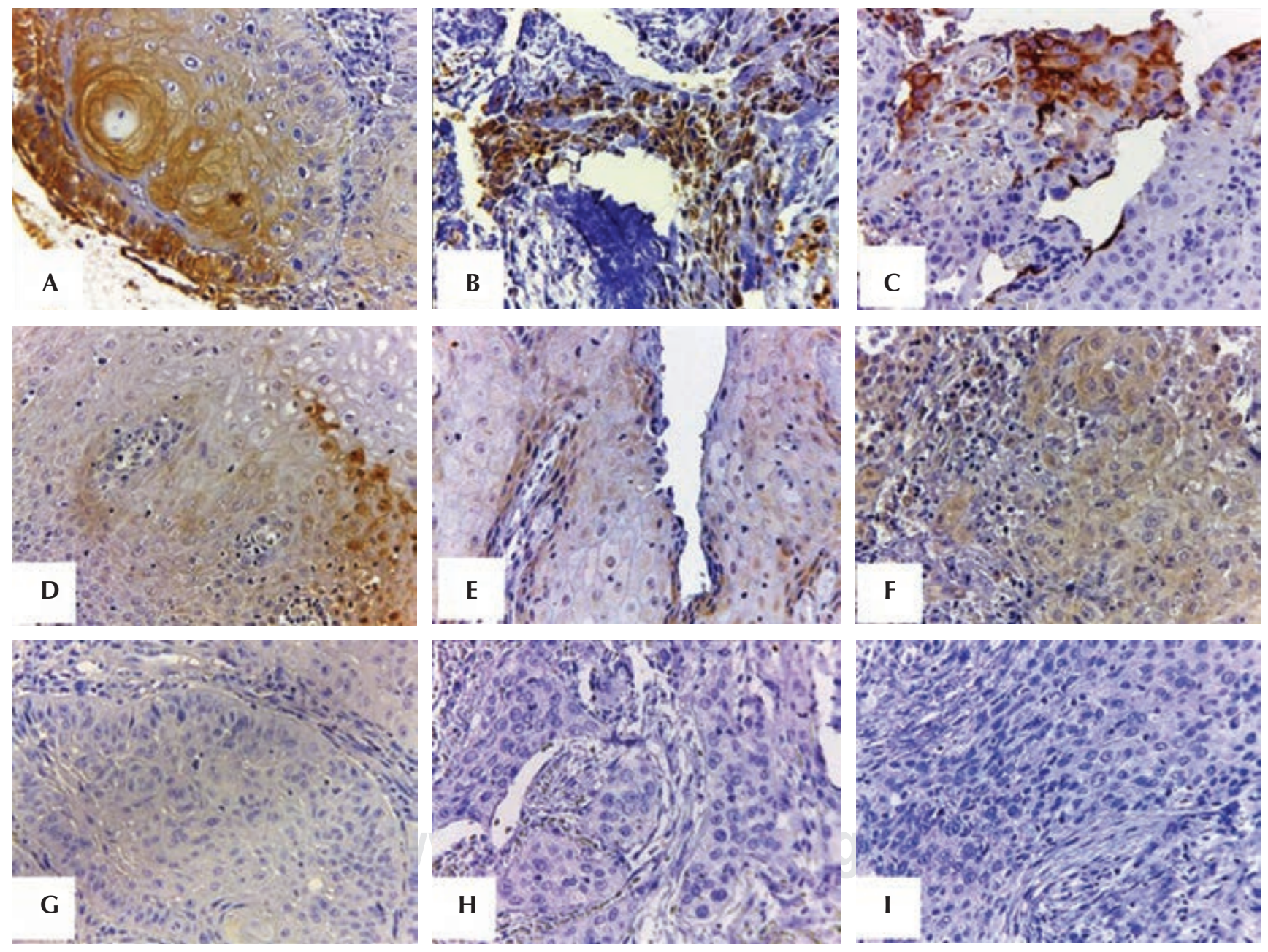

Figura 2: Inmunoexpresión de factores de crecimiento en carcinoma oral de células escamosas. A, B y C) VEGF en COCE bien, moderada y pobremente diferenciados, respectivamente. D, E y F) FGFR-1 en carcinomas bien, moderada y pobremente diferenciados. G) FGF-1 en carcinoma bien diferenciado. H) TGFB-1 en carcinoma moderadamente diferenciado. I) TGFBR-II en carcinoma pobremente diferenciado. 400 x. 
Tabla 2: Intensidad y proporción de inmunoexpresión (unidades ópticas de intensidad/gradificación de proporción).

\begin{tabular}{lccc} 
& $\begin{array}{c}\text { Bien } \\
\text { diferenciado }\end{array}$ & $\begin{array}{c}\text { Modera- } \\
\text { damente } \\
\text { diferenciado }\end{array}$ & $\begin{array}{c}\text { Pobremente } \\
\text { diferenciado }\end{array}$ \\
\hline VEGF & $16.7 / 3$ & $29.8 / 2$ & $32.3 / 2$ \\
FGFR-1 & $19.1 / 2$ & $8.6 / 3$ & $17.3 / 3$ \\
FGF-1 & 0 & 0 & 0 \\
TGFB-1 & 0 & 0 & 0 \\
TGFBR-ll & 0 & 0 & 0 \\
\hline
\end{tabular}

VEGF = factor de crecimiento vascular endotelial, FGFR-1 = receptor de factor de crecimiento fibroblástico-1, TGFB-1 = factor del crecimiento transformante beta-1, TGFBR-II = receptor del factor de crecimiento transformante beta-II.

molécula asociada a la angiogénesis y a otros eventos en la carcinogénesis es el receptor FGFR-1, el cual es el receptor del factor de crecimiento de fibroblastos. En nuestro análisis inmunohistoquímico se observó mayor expresión en los COCE bien diferenciados. Haq y colaboradores reportaron que la expresión de este receptor en los COCE es factible. ${ }^{27}$ La expresión de FGFR-1 se ha correlacionado con escasa diferenciación, mayor potencial de invasión y mal pronóstico en pacientes con COCE, además de influir en la transición epitelio-mesénquima. La expresión de FGFR-1 en fibroblastos en el frente invasivo de COCE se ha correlacionado con casos más invasivos, metástasis en ganglios linfáticos y de mal pronóstico. ${ }^{28}$ Ensayos in vitro han demostrado que inhibir esta molécula conduce a tasas disminuidas de proliferación e invasión celular, por lo cual podría ser candidata a terapias dirigidas en fases clínicas posteriores. ${ }^{29}$

En nuestro análisis de la inmunoexpresión de FGF-1, TGFB-1 y TGFBR-II su expresión fue negativa. Este hallazgo fue sumamente interesante, ya que diversos autores consideran estas moléculas fundamentales en la patogénesis del COCE. Por ejemplo, FGF-1 y miembros de la familia TGF se han vinculado con el desarrollo tumoral por un mecanismo autocrino, e inducción de la angiogénesis tumoral mediante un mecanismo paracrino. ${ }^{30,31}$

La angiogénesis es un proceso central para el desarrollo tumoral, por lo cual su medición ha sido un tema de investigación importante y recurrente. Ésta puede ser medida histológica (directa), radiológica (indirecta) y serológicamente con marcadores de actividad angiogénica (alternativa). La evaluación histológica es la más común y con ella se realiza la medición de la angiogénesis y la densidad microvascular (MVD). La MVD puede ser cuantificada en las áreas de mayor neovascularización, Ilamadas «focos calientes». ${ }^{15,16}$ La MVD se considera la herramienta más representativa y útil para evaluar los tejidos cancerosos. De hecho, numerosos estudios apoyan la hipótesis de que la MVD es un factor predictivo significativo para la progresión y el pronóstico de varios tipos de tumores malignos. ${ }^{17}$

La endoglina o CD105, que tiene un papel importante en la regulación de la angiogénesis, se requiere para la estructura e integridad normal de la vasculatura del adulto (Figura 3). Una de sus funciones es regular la migración de células endoteliales, favorecer la angiogénesis extraembrionaria normal y desarrollo del corazón embrionario. Puede regular los cambios de forma de las células endoteliales en respuesta al flujo sanguíneo, que impulsan la remodelación vascular y el establecimiento de la morfología vascular normal durante la angiogénesis.
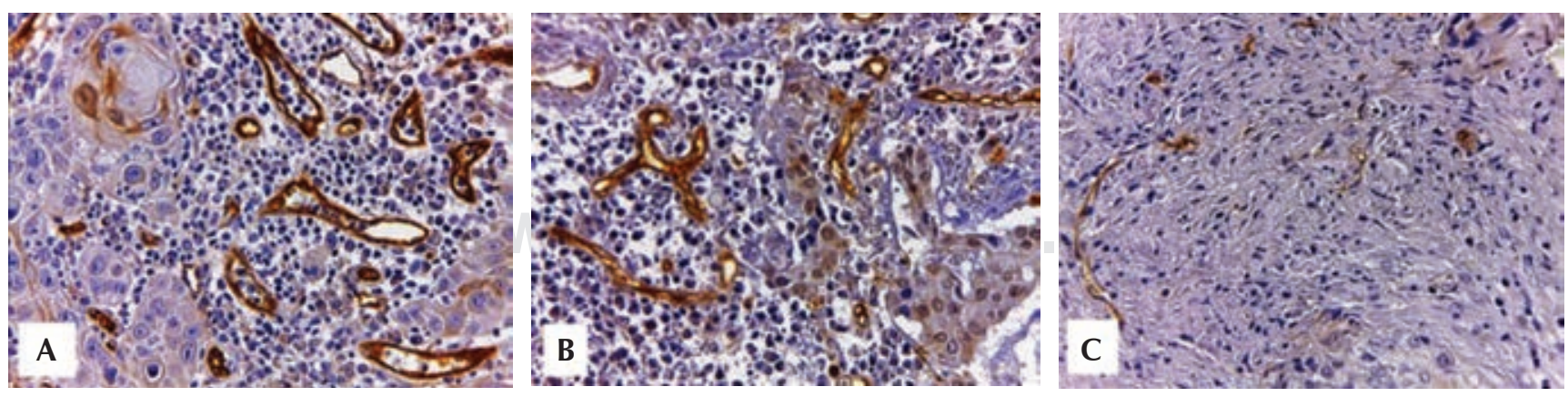

Figura 3: Inmunoexpresión de CD105 para determinar microdensidad vascular. A) Carcinoma bien diferenciado. B) Carcinoma moderadamente diferenciado. C) Carcinoma pobremente diferenciado. 400 x. 
Además puede actuar como correceptor de TGF-beta y participa en la cascada de señalización de TGF-beta/ BMP que finalmente conduce a la activación de factores de transcripción SMAD. ${ }^{32,33}$ La expresión de CD105 es una de las características más llamativas de los vasos sanguíneos recién formados, la cual se puede perder en vasos más maduros; ésta es una propiedad que puede ser explotada dentro del análisis de MVD. ${ }^{33}$ En nuestro análisis de la MVD observamos leve predominio del número de vasos en los carcinomas bien diferenciados seguidos de los pobre y moderadamente diferenciados sin mostrar ninguna diferencia significativa.

\section{CONCLUSIONES}

Aunque nuestra muestra fue reducida, estos resultados piloto nos sugieren que la angiogénesis es un fenómeno constante e independiente del grado de diferenciación; que la transformación formación de una neoplasia requerirá la formación de vasos sanguíneos nuevos durante todo su proceso patogénico y que este proceso puede ser modulado por factores de crecimiento tales como lo analizados en este trabajo. No obstante, nos deja interrogantes sobre cuál sería su relación con otras variables clínicas que han mostrado valor significativo en el pronóstico de los pacientes tal como la afección de nodo linfáticos o incluso en tamaño del tumor, gradificación TNM o estadio.

Investigación realizada gracias al programa UNAM-PAPIIT IN226720.

\section{BIBLIOGRAFÍA}

1. García CB, Gálvez MM, De la hoz RL. Acciones educativas sobre factores de riesgo del cáncer bucal en estudiantes de preuniversitario. Medicent Electron. 2019; 23 (3): 271-277.

2. Granados-García M, Arrieta-Rodríguez OG, Hinojosa-Gomez J. Tratamiento del cáncer: Oncología médica, quirúrgica y radioterapia. México: El Manual Moderno; 2016.

3. Sloan P. Squamous cell carcinoma. In: El-Naggar A, Chan J, Grandis J, Takata T, Slootweg J. WHO Classification of Head and Neck Tumours. 4ta ed. IARC: Lyon 2017.

4. Pérez CR, Cárdenas CE, Mondragón TP, Erazo VA. Biología molecular del cáncer y las nuevas herramientas en oncología. Rev Esp Med Quir. 2017; 22: 171-181.

5. GCO. Cancer today. [Consulted April 2020] Available in: https://gco. iarc.fr/today/online-analysis-table $\mathbf{v}=2018 \&$ mode $=$ cancer \&mode population $=$ continents \& population $=900 \&$ populations $=900 \&$ key $=$ asr \&sex $=0 \&$ cancer $=39 \&$ type $=2 \&$ statistic $=5 \&$ prevalen $\mathrm{ce}=1 \&$ population_group $=0$ \&ages_group $\% 5 \mathrm{~B} \% 5 \mathrm{D}=0$ \&ages group $\% 5 \mathrm{~B} \% 5 \mathrm{D}=1 \overline{7} \& \mathrm{nb}$ _items $=5 \&$ group_cancer $=1 \&$ include nmsc $=1$ \&include $n m s c$ other $=1$.
6. Gallegos HJ. El cáncer de cabeza y cuello. Factores de riesgo y prevención. Cir Ciruj. 2006; 74: 287-293.

7. Hanahan D, Weinberg RA. The hallmarks of cancer. Cell. 2000; 100 (1): 57-70.

8. Hanahan D, Weinberg RA. Hallmarks of cancer: the next generation. Cell. 2011; 144 (5): 646-674.

9. Stone WL, Varacallo M. Physiology, growth factor. In: StatPearls [Internet]. Treasure Island (FL): StatPearls Publishing; 2020-2018.

10. Pang $X$, Tang $Y L$, Liang $X H$. Transforming growth factor- $\beta$ signaling in head and neck squamous cell carcinoma: Insights into cellular responses. Oncol Lett. 2018; 16 (4): 4799-4806.

11. Lu SL, Reh D, Li AG, Woods J, Corless CL, Kulesz-Martin M, Wang $\mathrm{XJ}$. Overexpression of transforming growth factor $\beta 1$ in head and neck epithelia results in inflammation, angiogenesis, and epithelial hyperproliferation. Cancer Res. 2004; 64: 4405-4410.

12. Myoken Y, Myoken Y, Okamoto T, Sato JD, Takada K. Immunocytochemical localization of fibroblast growth factor-1 (FGF-1) and FGF-2 in oral squamous cell carcinoma (SCC). J Oral Pathol Med. 1994; 23 (10): 451-456.

13. Osada $A H$, Endo $K$, Kimura $Y$ et al. Addiction of mesenchymal phenotypes on the FGF/FGFR axis in oral squamous cell carcinoma cells. PLoS One. 2019; 14 (11): e0217451.

14. Ramjiawan RR, Griffioen AW, Duda DG. Anti-angiogenesis for cancer revisited: Is there a role for combinations with immunotherapy? Angiogenesis. 2017; 20 (2): 185-204. doi: 10.1007/s10456-017-9552-y.

15. Martínez EJ, Herrera LA. Angiogénesis: VEGF/VEGFRs como blancos terapéuticos en el tratamiento contra el cáncer. Cancerología. 2006; 1: 83-96.

16. Jiménez-Ríos MA, Solares-Sánchez ME. Angiogénesis en cáncer renal. Cancerología. 2006; 1: 113-121.

17. Miyata Y, Sakai H. Reconsideration of the clinical and histopathological significance of angiogenesis in prostate cancer: Usefulness and limitations of microvessel density measurement. Int J Urol. 2015; 22 (9): 806-815. doi: 10.1111/iju.12840.

18. Aviso de privacidad integral, Facultad de Odontología, Universidad Nacional Autónoma de México. [Consultado en agosto de 2020] Disponible en: http://www.odonto.unam.mx/sites/default/files/ inline-files/1.AP_Integral-Fac_Esc_Inst_Centros\%20\%20_0.pdf.

19. Trejo-Remigio DÁ, Jacinto-Alemán LF, Leyva-Huerta ER, NavarroBustos BR, Portilla-Robertson J. Ectodermal and ectomesenchymal marker expression in primary cell lines of complex and compound odontomas: a pilot study. Minerva Stomatol. 2019; 68 (3): 132-141. doi: 10.23736/S0026-4970.19.04166-9.

20. Kok SH, Chang HH, Tsai JY et al. Expression of Cyr61 (CCN1) in human oral squamous cell carcinoma: an independent marker for poor prognosis. Head Neck. 2010; 32 (12): 1665-1673. doi: 10.1002/hed.21381.

21. Villanueva-Sánchez FG, Leyva-Huerta ER, Gaitán-Cepeda LA. Cáncer en pacientes Jóvenes (Parte 1): Análisis clínico e histopatológico de carcinoma de células escamosas de la cavidad bucal en pacientes jóvenes. Un estudio descriptivo y comparativo en México. Odontoestomatología. 2016; 18 (27): 44-48.

22. Meza GG, Muñoz IJ, Páez VC, Cruz LB, Aldape BB. Carcinoma de células escamosas de cavidad bucal en un centro de tercer nivel de atención social en la Ciudad de México. Experiencia de cinco años. Av Odontoestomatol. 2009; 25 (1): 19-28.

23. Kademani D, Lewis JT, Lamb DH, Rallis DJ, Harrington JR. Angiogenesis and CD34 expression as a predictor of recurrence in oral squamous cell carcinoma. J Oral Maxillofac Surg. 2009; 67 (9): 1800-1805. doi: 10.1016/j.joms.2008.06.081. 
24. Konkimalla VB, Suhas VL, Chandra NR, Gebhart E, Efferth T. Diagnosis and therapy of oral squamous cell carcinoma. Expert Rev Anticancer Ther. 2007; 7 (3): 317-329.

25. O-charoenrat P, Rhys-Evans PH, Modjtahedi H, Eccles SA. The role of c-erbB receptors and ligands in head and neck squamous cell carcinoma. Oral Oncol. 2002; 38 (7): 627-640.

26. Astekar M, Joshi A, Ramesh G, Metgud R. Expression of vascular endothelial growth factor and microvessel density in oral tumorigenesis. J Oral Maxillofac Pathol. 2012; 16 (1): 22-26. doi: 10.4103/0973-029X.92968.

27. Haq F, Sung YN, Park I et al. FGFR1 expression defines clinically distinct subtypes in pancreatic cancer. J Transl Med. 2018; 16 (1): 374. doi: 10.1186/s12967-018-1743-9.

28. Mariz BALA, Soares CD, Morais TML, Fonseca FP, de Carvalho MGF, Jorge J. Expression of FGF-2/FGFR-1 in normal mucosa, salivary gland, preneoplastic, and neoplastic lesions of the oral cavity. J Oral Pathol Med. 2018; 47 (9): 816-822. doi: 10.1111/jop.12773.

29. Nguyen PT, Tsunematsu T, Yanagisawa S et al. The FGFR1 inhibitor PD173074 induces mesenchymal-epithelial transition through the transcription factor AP-1. Br J Cancer. 2013; 109 (8): 2248-2258. doi: 10.1038/bjc.2013.550.

30. Peralta-Zaragoza O, Lagunas-Martínez A, Madrid-Marina V. Factor de crecimiento transformante beta-1: estructura, función y mecanismos de regulación en cáncer. Salud Pública Méx. 2001; 43 (4): 340-351.

31. Sugerman PB, Joseph BK, Savage NW. Review article: the role of oncogenes, tumour suppressor genes and growth factors in oral squamous cell carcinoma: a case of apoptosis versus proliferation. Oral Dis. 1995; 1 (3): 172-188. doi: 10.1111/j.1601-0825.1995. tb00181.

32. Jamshidi S, Zargaran M, Baghaei F et al. An immunohistochemical survey to evaluate the expression of CD105 and CD34 in ameloblastoma and odontogenic keratocyst. J Dent (Shiraz). 2014; 15 (4): 192-198.

33. Kumar S, Ghellal A, Li C et al. Breast carcinoma: vascular density determined using CD105 antibody correlates with tumor prognosis. Cancer Res. 1999; 59 (4): 856-861.

\section{Correspondencia:}

Luis Fernando Jacinto-Alemán

E-mail: jacintoalemanlf@gmail.com

Conflicto de intereses: Los autores declaran no tener ningún conflicto de intereses. 